\title{
Partial coalescence of drops at liquid interfaces
}

\author{
FRANÇOIS BLANCHETTE* AND TERRY P. BIGIONI \\ The James Franck Institute, The University of Chicago, 5640 S. Ellis Ave., Chicago, Illinois 60637, USA \\ *e-mail: blanchef@uchicago.edu
}

$\mathbf{W}$ hen two separate masses of the same fluid are brought gently into contact, they are expected to fully merge into a single larger mass to minimize surface energy. However, when a stationary drop coalesces with an underlying reservoir of identical fluid, merging does not always proceed to completion ${ }^{1}$. Occasionally, a drop in the process of merging apparently defies surface tension by 'pinching off' before total coalescence occurs, leaving behind a smaller daughter droplet $^{1-3}$. Moreover, this process can repeat itself for subsequent generations of daughter droplets, resulting in a cascade of self-similar events ${ }^{2}$. Such partial coalescence behaviour has implications for the dynamics of a variety of systems, including the droplets in clouds ${ }^{4}$, ocean mist and airborne salt particles ${ }^{5}$, emulsions $^{6}$, and the generation of vortices near an interface ${ }^{7,8}$. Although it was first observed almost half a century ago, little is known about its precise mechanism. Here, we combine highspeed video imaging with numerical simulations to determine the conditions under which partial coalescence occurs, and to reveal a dynamic pinch-off mechanism. This mechanism is critically dependent on the ability of capillary waves to vertically stretch the drop by focusing energy on its summit.

Liquid drops were deposited from a pipette onto the surface of an identical liquid in air, and filmed with a high-speed digital camera. When drops were deposited gently, they rested on the surface until the interstitial air layer drained ${ }^{9}$. The two liquid masses then touched to form a thin liquid bridge that rapidly widened, on a timescale ranging from 10 to $200 \mu$ s depending on the drop size, sending capillary waves racing up the drop surface. As the drop drained into the reservoir, its shape generally evolved into a cylindrical column with a height greater than that of the initial drop. The neck then narrowed due to the inward pull of surface tension, and under the appropriate conditions, pinched off to form a daughter droplet with a radius approximately half that of the initial drop ${ }^{1,2}$ (Fig. 1 and Supplementary Information Video S1). After pinch-off, the daughter droplet bounced a number of times, and could reside on the interface ${ }^{10,11}$ for up to a second before coalescing with the lower fluid. This process could then repeat to leave behind a smaller droplet, a sequence of events referred to as a coalescence cascade ${ }^{2}$.

To gain insight into the mechanism behind partial coalescence, we numerically simulated the coalescence process. We solved the
Navier-Stokes equations, including surface tension as a force localized on the interface ${ }^{12,13}$, to track the time-evolution of the drop shape, as well as the pressure and velocity fields inside the drop. Starting from a stationary drop surrounded by air, and connected to the underlying liquid layer by a narrow neck, the system was allowed to evolve until the drop either fully coalesced or pinched off into a daughter droplet. Directly comparing computations and experiments shows that our simulations accurately captured the evolution of the drop shape (Fig. 1 and Supplementary Information Video S2). Moreover, for small drops, for which the effect of gravity is negligible compared with capillary effects, general features of the flow such as the ratio of mother to daughter drop radii $(\sim 0.55)$ and the time elapsed between the onset of coalescence and pinch-off $\left(\sim 1.7\left(R^{3} \rho / \sigma\right)^{1 / 2}\right.$, where $R$ is the drop radius, $\sigma$ the surface tension, and $\rho$ is the density of the liquid) agree well with published data ${ }^{2,14}$.

Mechanisms for the generation of daughter droplets have been postulated, but have never been confirmed ${ }^{1,15}$. It has been suggested $^{1}$ that pinch-off results from a static Rayleigh-Plateau instability ${ }^{16}$, which causes a thin column of fluid to break up into a sequence of droplets under the influence of surface tension. This instability was thought to occur when the coalescing drop evolved into a roughly cylindrical shape, a notion difficult to verify experimentally. We tested this hypothesis by carrying out the following computation: for flow parameters that normally exhibited pinch-off, we interrupted the evolution of the interface in its maximally stretched state (Fig. 1e), set all velocities to zero, and then restarted the simulations (Fig. 2). This process never led to the formation of a daughter droplet, as the geometry of the drop was not sufficiently elongated. The Rayleigh-Plateau instability is therefore not the cause of the formation of a daughter droplet, which suggests that the pinch-off mechanism depends on the early dynamics of coalescence.

By examining the velocity fields (for example, Fig. 1e), we discovered that pinch-off critically depends on the inward momentum of the collapsing neck. When gravitational effects are negligible, the coalescence process is in fact controlled by a competition between the vertical and horizontal rates of collapse, both of which are driven by surface tension. The downward pull of surface tension at the drop's summit, where the curvature is maximal, is in general larger than the inward, horizontal pull of the 

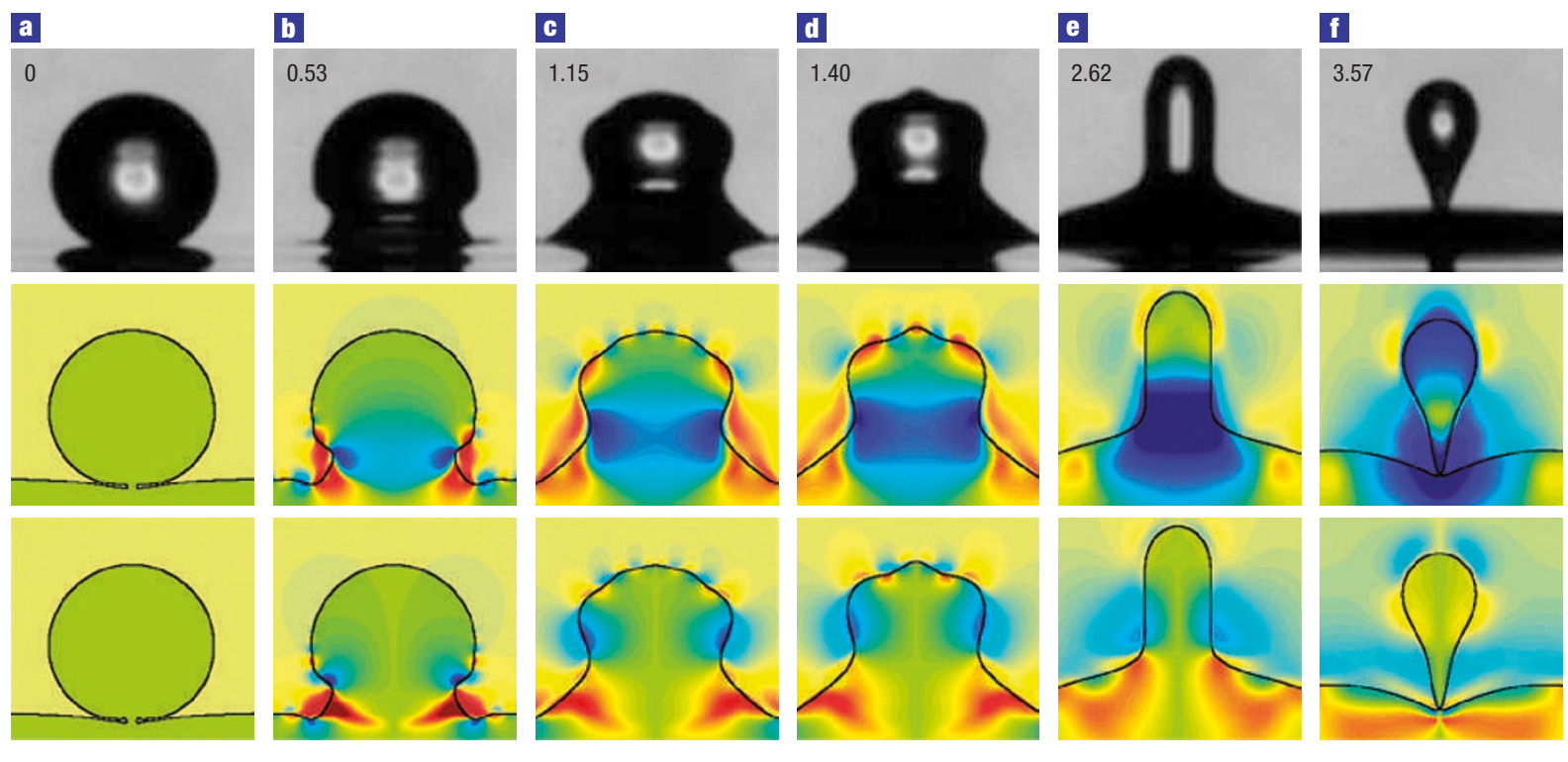

Figure 1 Partial coalescence of an ethanol drop. Experimental (top row) and numerical (middle and bottom rows) images show the evolution of a partially coalescing drop of ethanol, with initial radius $0.535 \mathrm{~mm}$ and beginning at rest on an ethanol reservoir $\left(0 h=\mu /(\rho \sigma R)^{1 / 2}=0.011, B o=g \rho R^{2} / \sigma=0.09\right)$. Times are given in milliseconds, and are the same for the experimental and numerical images in each column. Numerical simulations were carried out with modified air parameters, but other parameters are identical to the experimental values. We show the vertical velocity (middle row) with red and blue indicating upward and downward motion, respectively, and the horizontal velocity (bottom row) with red and blue indicating motion away from and towards the centre, respectively. Velocities in the gas use the same colour scheme, but are shown paler to better differentiate the fluids. The experimental time $t=0.00 \mathrm{~ms}$ cannot be accurately determined from the experimental pictures, so was chosen to best synchronize the experimental and numerical time sequences.

neck. This leads us to expect the vertical collapse to prevail, as in the static case. The horizontal collapse may, therefore, only induce pinch-off if the vertical collapse is sufficiently retarded. Such a delay is achieved by the convergence of capillary waves that are generated by the opening of the neck in the early stages of coalescence. These waves travel up the side of the drop, and carry enough momentum to significantly distort the drop as they converge on its summit. This convergence of capillary waves can then stretch the drop upwards by as much as $30 \%$ of its initial radius (Fig. 3). If the waves are sufficiently vigorous to counteract the vertical collapse, the horizontal collapse is then able to reach completion, producing a daughter droplet.

Simulations also revealed that the viscosity of the liquid determines the extent to which the capillary waves can stretch the drop and delay vertical collapse. For sufficiently viscous liquids, capillary waves are strongly damped, and little momentum reaches the top of the drop, leading to complete coalescence. We characterize the damping of the waves by comparing a typical damping time with the time needed for the capillary waves to reach the top of the drop. For capillary waves with a wavelength much smaller than the drop radius, the wave velocity ${ }^{17}$ is $v_{\mathrm{w}}=(\sigma k / \rho)^{1 / 2}$, where $k$ is the wavenumber. We therefore estimate that waves take a time $t=\pi R /(\sigma k / \rho)^{1 / 2}$ to reach the top of the drop. Approximating the damping rate ${ }^{17}$ by $D=2 \mu k^{2} / \rho$, where $\mu$ is the liquid viscosity, a condition for a significant amount of momentum to reach the top of the drop is $D t<1$. Equivalently, this condition may be written as $2 \pi(R k)^{3 / 2} \mathrm{Oh}<1$, where the Ohnesorge number, $O h=\mu /(\rho \sigma R)^{1 / 2}$, is a non-dimensionalized viscosity that is the ratio of typical viscous and surface forces. Note that if we use $v=(\sigma / \rho R)^{1 / 2}$ as a typical velocity, then $O h$ corresponds to the inverse Reynolds number.

The above argument suggests that a critical Ohnesorge number, $O h^{*}$, should exist, above which pinch-off does not occur. As

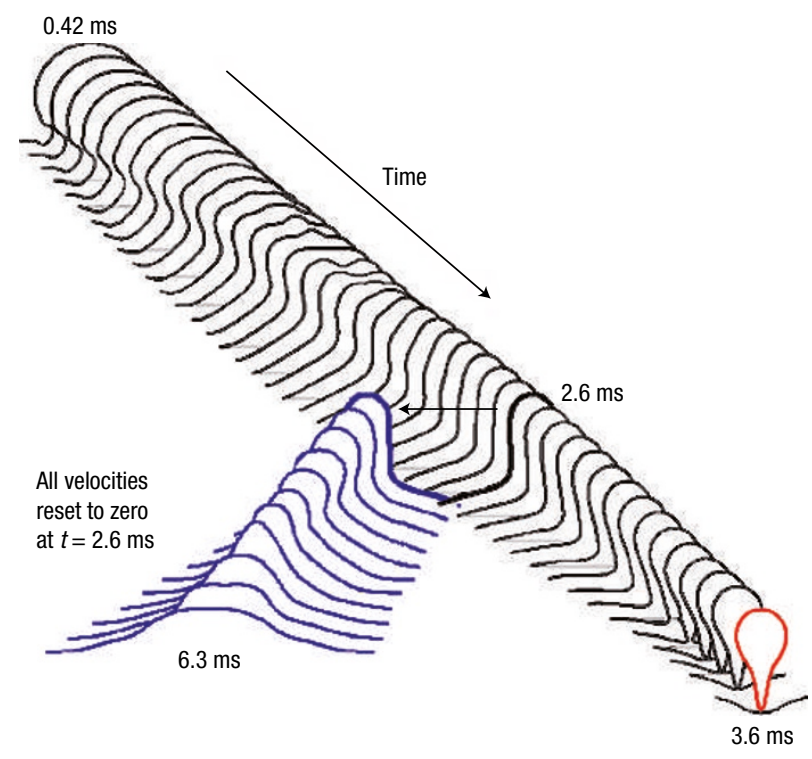

Figure 2 Time evolution of a pinching drop. The computed evolution of the interface of a drop of ethanol coalescing with a reservoir of ethanol (black curves, $0.082 \mathrm{~ms}$ apart). The parameters are identical to those used in Fig. 1. Resetting the fluid velocity to zero at the maximally stretched state (Fig. 1e) yielded no pinch-off, showing that the static Rayleigh-Plateau instability is not responsible for the formation of a daughter droplet (blue curves, $0.24 \mathrm{~ms}$ apart).

$R k$ generally exceeds unity, we expect $O h^{*}$ to be significantly smaller than one. Our simulations indicate that an $\mathrm{Oh}^{*}$ does 


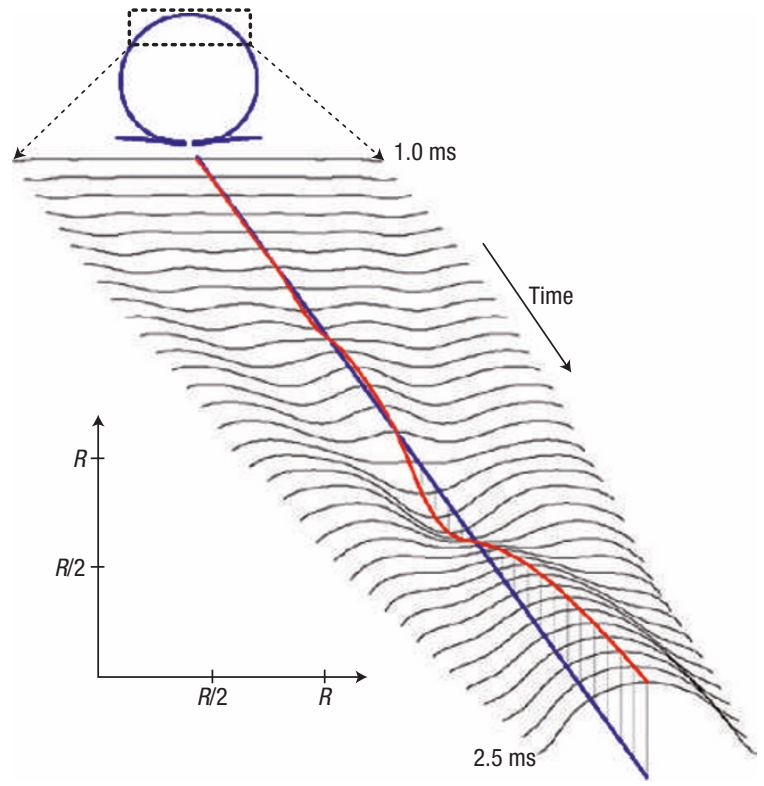

Figure 3 Capillary waves converge to stretch the drop. The computed evolution of the vertical displacement of the interface, relative to its initial position, shows capillary waves converging from each side to stretch the drop vertically (curves are $0.041 \mathrm{~ms}$ apart). Only the top portion of the drop is shown, as highlighted by the dashed box. The red curve shows the oscillatory motion of the drop's summit with respect to its initial position, indicated by the blue line. The axes show the scale of the initial drop radius, $R$, and indicate that the upward displacement of the summit can exceed $0.3 R$

indeed exist, and we show in Fig. 4 its dependence on the Bond number, $B o=g \rho R^{2} / \sigma$, a non-dimensionalized weight that compares gravitational and surface forces. The critical Ohnesorge number shows a weak dependence on gravitational effects, and was found to be $0.026 \pm 0.001$ when gravity was negligible.

Experimental measurements were made on ethanol/water and ethanol/glycerin solutions ${ }^{18}$ to vary surface tension and viscosity. The results of our experiments are in good agreement with our computational results, and show that $O h^{*}=0.026 \pm 0.003$ at low Bond numbers. Over a broad range of Bond numbers, the experimental data show a transition between partial and full coalescence that is bounded by computational results obtained starting from two sets of idealized initial conditions: a spherical drop on a planar interface, and a drop at equilibrium on an interface, deformed together under gravity. Under non-idealized conditions, we found numerically that the value of $\mathrm{Oh}^{*}$ was only weakly affected by fluid motion inside the drop, but was sensitive to variations in the position of the drop at the onset of coalescence (for example, due to bouncing or oscillating). The value of $O h^{*}$ varied by up to $25 \%$ when we varied the initial height of the drop by as little as $0.1 R$ from the equilibrium position.

When a sequence of partial coalescence events occurs, the ratio of viscous to surface forces increases as the drop size decreases with each successive coalescence event. Eventually $\mathrm{Oh}^{*}$ is exceeded, at which point the drop fully merges with the reservoir. The size and composition of a drop uniquely determine the values of $\mathrm{Oh}$ and Bo; Fig. 4 therefore allows us to predict whether or not a daughter droplet will be generated from any mother drop. For ethanol, the smallest drop observed to pinch-off had a radius of $97 \pm 5 \mu \mathrm{m}$ compared with a predicted minimum radius of $98 \mu \mathrm{m}$; for water,

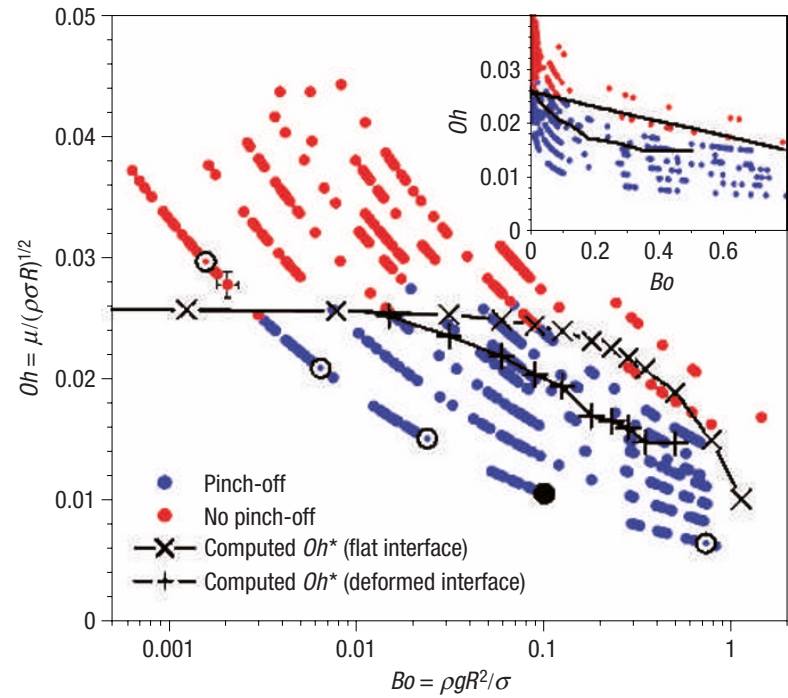

Figure 4 Phase diagram for partial and total coalescence. Experimental results of coalescence events are plotted in terms of the Bond number, a non-dimensionalized weight and a measure of sagginess, and the Ohnesorge number, a non-dimensionalized viscosity. The blue dots indicate observations of drops that did pinch-off, whereas the red dots indicate drops that merged completely with the lower fluid. The black circles correspond to a sequence of daughter droplets observed when an ethanol drop repeatedly underwent partial coalescence, where the drop size decreases up and to the left. The filled black circle corresponds to Fig. 1. Numerically computed values of the critical Ohnesorge number, $O h^{*}$, are also shown for two sets of initial conditions: a spherical drop on a planar surface $(x)$ and a drop at equilibrium on an interface, deformed together under gravity $(+)$. The error bars increase up and to the left, but most are smaller than the data markers. A sample error bar is shown.

the minimum radius for which a drop may pinch off is estimated to be $22 \mu \mathrm{m}$.

We have shown that partial coalescence of liquid drops is a dynamically driven process, and does not result from the RayleighPlateau instability. The occurrence of pinch-off is determined by a competition between the vertical and horizontal collapses of the connected drop. When the vertical collapse is sufficiently delayed by converging capillary waves, the horizontal collapse succeeds in pinching off the neck and producing a daughter droplet. However, if the waves are strongly damped before converging on the summit, so that the drop is not sufficiently stretched, the drop will fully coalesce with the underlying fluid. The phase boundary between partial and total coalescence is characterized by the critical Ohnesorge number, which depends weakly on the Bond number. When gravitational effects are negligible, we found $\mathrm{Oh}^{*}$ to be approximately 0.026 .

If the surrounding gas is replaced by a second fluid, simulations show that pinch-off occurs through the same mechanism, but $\mathrm{Oh}^{*}$ depends on the density and viscosity ratios of the two liquids. The coalescence of two drops of different sizes also resulted in partial coalescence in fluids with a viscosity comparable to that of water, provided the drops had radii with a ratio of 2 or larger. Drops that were significantly deformed under gravity $(B o>0.2)$, exhibited more complex dynamics: in experiments, satellite droplets were occasionally observed to form between the daughter droplet and the interface ${ }^{1,2}$, and tiny droplets were ejected ${ }^{19}$ from the top of the mother drop when particularly vigorous capillary waves converged on its summit. Our study also suggests that the presence 
of surfactants, which typically enhances wave damping ${ }^{20}$, should inhibit partial coalescence.

\section{METHODS}

Experiments were carried out on ethanol solutions ${ }^{18}$ containing either $0-40 \%$ water or $20-30 \%$ glycerin, to vary surface tension and viscosity while keeping surface tension low to minimize contamination. Solutions were frequently discarded to avoid possible Marangoni effects resulting from evaporation or contamination of the solutions. Photographs were taken with a Phantom V7 high-speed video camera, at a rate of 47,000 fps.

We numerically solved the Navier-Stokes equations using a staggered grid, exploiting the axial symmetry for computational efficiency ${ }^{21}$, and including surface tension as a force localized on the interface ${ }^{12,13}$. We computed the pressure and velocity fields inside the drop, and tracked the interface using markers that moved with the fluid. No-slip boundary conditions were used on the top, bottom and side walls; these boundaries were sufficiently far to have no influence on the dynamics of motion. Our simulations were tested for numerical convergence, as well as mass and energy conservation. Simulations showed the formation of a vortex ring in the lower fluid ${ }^{22}$, and accurately captured the evolution of the drop shape (Fig. 1). Excellent agreement with experiments was observed despite the need to overestimate the air density and viscosity ( $\rho_{\text {air }}=0.05 \mathrm{~g} \mathrm{~cm}^{-3}, \mu_{\text {air }}=0.05 \mathrm{cP}$ ) for numerical convergence, and to overestimate the initial drop-interface gap thickness $(100 \mu \mathrm{m})$ to avoid the need for very high spatial resolution associated with the separation of length scales between the drop and gap sizes. Other parameters were identical to the experimental values (for example, $\rho_{\text {liq }}=0.786 \mathrm{~g} \mathrm{~cm}^{-3}, \mu_{\text {liq }}=1.05 \mathrm{cP}$, $\sigma=22$ dynes $\mathrm{cm}^{-1}$ for ethanol).

Received 28 November 2005; accepted 3 March 2006; published 1 April 2006.

\section{References}

1. Charles, G. E. \& Mason, S. G. The mechanism of partial coalescence of liquid drops at liquid/liquid interfaces. J. Colloid Sci. 15, 105-122 (1960).

2. Thoroddsen, S. T. \& Takehara, K. The coalescence cascade of a drop. Phys. Fluids 12, $1265-1267$ (2000).

3. Charles, G. E. \& Mason, S. G. The coalescence of liquid drops with flat liquid/liquid interfaces. J. Colloid Sci. 15, 236-267 (1960).
4. Berry, E. X. \& Reinhardt, R. L. Analysis of cloud drop growth by collection. 3. Accretion and self-collection. J. Atmos. Sci. 31, 2118-2126 (1974).

5. Raes, F. et al. Formation and cycling of aerosols in the global troposphere. Atmos. Environ. 34, $4215-4240$ (2000)

6. Bhakta, A. \& Ruckenstein, E. Decay of standing foams: drainage, coalescence and collapse. Adv. Colloid Interface Sci. 70, 1-124 (1997).

7. Thompson, J. J. \& Newall, H. F. On the formation of vortex rings by drops falling into liquids and some allied phenomena. Proc. R. Soc. Lond. 39, 417-436 (1885).

8. Sarpkaya, T. Vorticity, free surface, and surfactants. Ann. Rev. Fluid Mech. 28, 83-128 (1996).

9. Reynolds, O. On the floating of drops on the surface of water depending only on the purity of the surface. Proc. Lit. Phil. Soc. Manchester 21, 1-2 (1881).

10. Couder, Y., Fort, E., Gautier, C. H. \& Boudaoud, A. From bouncing to floating: Noncoalescence of drops on a fluid bath. Phys. Rev. Lett. 94, 177801 (2005)

11. Couder, Y., Protiere, S., Fort, E. \& Boudaoud, A. Dynamical phenomena: Walking and orbiting droplets. Nature 437, 208 (2005).

12. Lafaurie, B., Nardone, C., Scardovelli, R., Zaleski, S. \& Zanetti, G. Modelling merging and fragmentation in multiphase flows with SURFER. J. Comput. Phys. 113, 134-147 (1994).

13. Popinet, S. \& Zaleski, S. A front tracking algorithm for the accurate representation of surface tension. Int. J. Numer. Methods Fluids 30, 775-793 (1999).

14. Bach, G. A., Koch, D. L. \& Gonipath, A. Coalescence and bouncing of small aerosol droplets. J. Fluid Mech. 518, 157-185 (2004).

15. Pikhitsa, P. \& Tsargorodskaya, A. Possible mechanism for multistage coalescence of a floating droplet on the air/liquid interface. Colloids Surf. A 167, 287-291 (2000).

16. Plateau, J. A. F. Statique Expirimentale et Thiorique des Liquides Soumis aux Seules Forces Moliculaires (Gauthier-Villars, Paris, 1873).

17. Landau, L. D. \& Lifshitz, E. M. Fluid Mechanics 2nd edn (Pergamon, New York, 1987)

18. Ernst, R. C., Watkins, C. H. \& Ruwe, H. H. The physical properties of ternary systems ethyl alcohol-glycerin-water. J. Phys. Chem. 40, 627-635 (1936).

19. Renardy, Y. et al. Pyramidal and toroidal water drops after impact on a solid surface. J. Fluid Mech. 484, 69-83 (2003).

20. Asaki, T. J., Thiessen, D. B. \& Marston, P. L. Effect of an insoluble surfactant on capillary oscillations of bubbles in water: Observation of a maximum in the damping. Phys. Rev. Lett. 75, 2886-2889 (1995).

21. Popinet, S. \& Zaleski, S. Bubble collapse near a solid boundary: a numerical study of the influence of viscosity. J. Fluid Mech. 464, 137-163 (2002).

22. Shankar, P. N. \& Kumar, M. Vortex rings generated by drops just coalescing with a pool. Phys. Fluids 7, 737-746 (1995).

\section{Acknowledgements}

The authors gratefully acknowledge W. W. Zhang and H. M. Jaeger for helpful discussions, as well as E. I. Corwin for stimulating exchanges. This work was supported by the NSF through the MRSEC program No. DMR-0213745.

Correspondence and requests for materials should be addressed to F.B.

Supplementary Information accompanies this paper on www.nature.com/naturephysics.

\section{Competing financial interests}

The authors declare that they have no competing financial interests.

Reprints and permission information is available online at http://npg.nature.com/reprintsandpermissions/ 